\title{
Composición Factorial del Cuestionario de Estilos Atributivos Área de Logros Académicos en Universitarios Mexicanos
}

\author{
Perla J. Jurado, Humberto Blanco, María del Carmen Zueck y Jesús E. Peinado* \\ Universidad Autónoma de Chihuahua. Facultad de Ciencias de la Cultura Física. DES Salud CA 101. \\ Calle Escorza 900, CP 31000 Chihuahua, Chih.- México. (e-mail: pjurado@uach.mx, hblanco@uach.mx, \\ mzueck@uach.mxy jpeinad@uach.mx)
}

* Autor a quien debe ser dirigida la correspondencia

Recibido Abr. 28, 2016; Aceptado Jun. 7, 2016; Versión final Jul. 10, 2016, Publicado Dic. 2016

\begin{abstract}
Resumen
Se analiza la composición factorial del Cuestionario de Estilos Atributivos en el área de logros académicos en universitarios mexicanos La muestra fue de 1469 alumnos de las licenciaturas de Educación Física y Motricidad Humana que se ofrecen en la Universidad Autónoma de Chihuahua en México, con una edad media de 20.67 años. A través de análisis factoriales confirmatorios se obtuvo una estructura de cuatro factores. La estructura de cuatro factores (atribución a la suerte, atribución al esfuerzo, atribución a la habilidad y atribución al profesor) ha mostrado adecuados indicadores de ajuste de fiabilidad y validez. Además, los resultados de los análisis factoriales llevados a cabo con las submuestras, indican la existencia de fuertes evidencias de la estabilidad de la estructura factorial.
\end{abstract}

\section{Factor structure of the Questionnaire of Attributional styles Area of Academic Achievement in Mexican university students}

\begin{abstract}
The factor structure of the Questionnaire of Attributional styles of the area of academic achievement in Mexican university students is analyzed. The sample consisted of 1469 participants, all of them students of the degree of Physical Education and Human Motricity offered at the Autonomous University of Chihuahua in Mexico, with a mean age of 20.67 years. Through confirmatory factor analysis a four-factor structure was obtained. The structure of the four factors (attribution to luck, attribution to effort, attribution to skill and attribution to teacher) showed adequate indicators of adjustment of reliability and validity. In addition, factor analysis conducted with subsamples indicated the presence of strong evidence of factor structure stability.
\end{abstract}

Keywords: attributional styles; factor structure; construct validation; structural equation 


\section{INTRODUCCIÓN}

Uno de los principales problemas de la juventud hoy en día es la pérdida de motivación en las actividades académicas, carecen de voluntad para llevarlas a cabo, lo que a su vez se traduce en un rendimiento académico bajo (Fernández, Arnaiz y Barca, 2015). Por ello, existe un interés creciente en el ámbito educativo de conocer los factores cognitivos y conductuales que contribuyan o dificulten el desempeño académico del estudiante (Blanco et al., 2013; Camgoz et al., 2008; Soria-Barreto y Zúñiga-Jara, 2014). De acuerdo a la teoría atribucional es importante comprender y distinguir las características relacionadas con la motivación que evidencian los estudiantes cuando se enfrentan a un ambiente de aprendizaje, que conozcan cuáles son las causas a las que asocian sus resultados académicos y que al mismo tiempo hagan uso de estrategias que les permitan un adecuado proceso de aprendizaje (Durán-Aponte y Pujol, 2012, 2013; Inglés et al., 2012; Mkumbo y Amani, 2012; Weiner, 2000).

Por otro lado, el ser humano como tal, siempre está en constante búsqueda de las causas y el porqué de su comportamiento y el de otros (Mkumbo y Amani, 2012); de ahí la importancia de los estilos de atribución, ya que son precisamente éstos los que describen la forma en que un individuo conceptualiza habitualmente la causa de los acontecimientos, son las interpretaciones que cada persona le da a su realidad, es decir, el modo de explicar la causalidad; donde, de acuerdo a los estilos de atribución algunos refieren sus éxitos o fracasos a causas internas y otros a causas externas (Salvador y Mayoral, 2011; Tavakolizadeh y Ebrahimi, 2011; Weiner, 2000, 2004).

Con base en el meta análisis reportado por Richardson et al. (2012) sobre los correlatos psicológicos y el rendimiento académico en estudiantes universitarios se ha evidenciado que los estilos de atribución tienen un efecto en el rendimiento académico, donde algunos estudiantes tienden a explicar las bajas calificaciones basados en sus propias deficiencias, es decir, debido a causas internas tales como la falta de esfuerzo o habilidad, mientras que otros lo atribuyen a causas externas como la mala suerte 0 a una enseñanza insuficiente.

Además de acuerdo a Salvador y Mayoral (2011) esas interpretaciones que se hacen de las conductas afectan la autoimagen y como consecuencia la motivación y en el comportamiento de las personas (Salvador y Mayoral, 2011; Sanjuán y Magallares, 2006). De ahí la importancia de contar con herramientas de evaluación adecuadas y de probar sus características psicométricas con el fin de aportar nuevas evidencias y contribuir en la adaptación de éstas (Brenlla y Vázquez, 2010; Durán-Aponte y Pujol, 2013; Ferrando et al., 2011; Inglés et al., 2008).

Existe una gran variedad de instrumentos para medir estilos de atribución, sin embargo, hay pocos enfocados al área académica y la mayoría son usados en estudiantes de primaria y secundaria; entre ellos se encuentra el de Alonso y Sánchez (1992) el cual mide dos dimensiones, la primera es logros académicos con 7 escalas: 1. Atribución del éxito académico a la buena suerte, hace referencia a la atribución de los éxitos a causas externas al propio sujeto no controlables por él, como la suerte y otras personas. 2. Atribución del fracaso académico a la falta de esfuerzo, hace referencia a la falta de esfuerzo, causa normalmente percibida como interna, variable y controlable. 3. Atribución del éxito académico a la habilidad, el éxito se atribuye a causas internas, estables y controlables. 4. Atribución del fracaso al profesor, se percibe como una causa externa y no controlable. 5. Atribución del éxito al esfuerzo, hace referencia a una causa habitualmente percibida como interna, variable y controlable. 6 . Atribución del fracaso académico a la mala suerte, se refiere a una causa percibida como externa, variable y no controlable. 7 . Atribución del fracaso a la falta de habilidad, se refiere a una causa percibida como interna, estable y no controlable; y la segunda dimensión es relaciones interpersonales con 5 escalas: 1. Internalización del fracaso en las relaciones interpersonales. 2. Atribución del éxito en las relaciones interpersonales al esfuerzo. 3. Externalización del éxito en las relaciones interpersonales. 4. Atribución del éxito en las relaciones interpersonales a la habilidad y 5 . Externalización del fracaso.

En cuanto a los estudios de validez del Cuestionario de Estilos Atributivos Alonso y Sánchez (1992) reportaron que los siete factores explican el $44.99 \%$ de la varianza mientras que el coeficiente alpha de Cronbach de las siete escalas del área de logros académicos oscila entre .650 y .779 . Por su parte Matalinares et al. (2009) reportaron una validez del instrumento mediante el coeficiente $\mathrm{V}$ de Aiken y en referencia a la confiabilidad del cuestionario encontraron correlaciones ítem-test que oscilan entre 0.25 y 0.50, con un alpha de Cronbach de .662 .

Por tanto, el presente estudio clasificado como de corte instrumental (Montero y León, 2007) se ha dirigido a proporcionar apoyo empírico a la división factorial del Cuestionario de Estilos Atributivos en el Área de Logros académicos en la versión de la adaptación hecha por Matalinares et al. (2009), aunque el instrumento consta de dos áreas sólo se analizará la de logros académicos en este trabajo; esto ante la 
necesidad de comprobar la estructura factorial de un instrumento y la equivalencia psicométrica del mismo en distintos grupos; ya que en el contexto de la comparación intergrupal, es indispensable plantearse la necesidad de llevar a cabo la adaptación de un instrumento de medida psicológica que cumpla con todos los criterios de equivalencia, pero sobre todo establecer si la misma estructura factorial es aplicable a distintos grupos de sujetos o, en general a distintas poblaciones (Abalo et al., 2006).

\section{METODOLOGÍA}

Se describen quienes son los participantes, luego se dan detalles del instrumento usado (un cuestionario) y se explica el procedimiento de colección de datos, para luego describir como se realiza el análisis de dichos datos

\section{Participantes}

La muestra de 1469 participantes (772 mujeres y 697 hombres) se obtuvo mediante un muestreo por conveniencia, tratando de abarcar la representatividad de las diferentes licenciaturas que se ofrecen en la Facultad de Ciencias de la Cultura Física de la Universidad Autónoma de Chihuahua. La edad de los participantes fluctuó entre los 18 y 28 años, con una media de 20.67 y una desviación estándar de 1.90 años. La muestra fue aleatoriamente dividida en dos partes utilizando el Statistical Package for the Social Sciences (SPSS) en su versión 18.0; con el fin de realizar estudios paralelos que permitieran corroborar y verificar los resultados obtenidos (validación cruzada). La submuestra 1 quedó constituida por 763 participantes (399 mujeres y 364 hombres). Las edades fluctúan entre los 18 y 28 años, con una media de 20.68 y una desviación estándar de 1.99 años. La submuestra 2 quedó compuesta por 706 participantes (373 mujeres y 333 hombres). Las edades fluctúan entre los 18 y 28 años, con una media de 20.65 y una desviación estándar de 1.80 años.

\section{Instrumento}

El Cuestionario de Estilos Atributivos en el Área de Logros académicos adaptado por Matalinares et al. (2009) consta de 39 ítems tipo Likert que evalúan los patrones atributivos a través de afirmaciones sobre las causas del éxito y el fracaso en distintas situaciones por medio de siete factores: 1 . Atribución del éxito académico a la buena suerte, hace referencia a la atribución de los éxitos a causas externas al propio sujeto no controlables por él, la suerte y otras personas. 2. Atribución del fracaso académico a la falta de esfuerzo, hace referencia a la falta de esfuerzo, causa normalmente percibida como interna, variable y controlable. 3. Atribución del éxito académico a la habilidad, el éxito se atribuye a causas internas, estables y controlables. 4. Atribución del fracaso al profesor, se percibe como una causa externa y no controlable. 5. Atribución del éxito al esfuerzo, hace referencia a una causa habitualmente percibida como interna, variable y controlable. 6. Atribución del fracaso académico a la mala suerte, se refiere a una causa percibida como externa, variable y no controlable. 7. Atribución del fracaso a la falta de habilidad, se refiere a una causa percibida como interna, estable y no controlable. Para fines de esta investigación se hicieron tres adaptaciones a la versión de Matalinares y colaboradores, la primer adaptación consistió en utilizar una escala de respuesta de 0 a 10 donde 0 es totalmente en desacuerdo y 10 totalmente de acuerdo; esta adaptación se justifica en cuanto a que los participantes están acostumbrados a la escala de 0 a 10, ya que así han sido evaluados por el sistema educativo de nuestro país (México). Viciana et al. (2007) reportan un cambio similar en la validación de una escala con población española. La segunda adaptación radicó en cambiar algunos términos utilizados en los ítems de la versión original con el fin de utilizar un lenguaje más adecuado al contexto de la cultura mexicana. La tercera adaptación consistió en aplicar el instrumento por medio de una computadora con el fin de permitir el almacenamiento de los datos sin etapas previas de codificación, con una mayor precisión y rapidez. Además el sentido de los ítems se giró hacia el control interno.

\section{Procedimiento}

Se invitó a participar en el estudio a los alumnos de las licenciaturas que se ofrecen en la Facultad de Ciencias de la Cultura Física (FCCF) de la Universidad Autónoma de Chihuahua. Los que aceptaron participar firmaron la carta de aceptación correspondiente. Luego se aplicó el instrumento, antes descrito, por medio de una computadora personal (módulo administrador del instrumento del editor de escalas de ejecución típica), en una sesión de aproximadamente 40 minutos; en los laboratorios o centros de cómputo de la FCCF. Al inicio de cada sesión se hizo una pequeña introducción sobre la importancia de la investigación y de cómo acceder al instrumento. Se les solicitó la máxima sinceridad y se les garantizó la confidencialidad de los datos que se obtuvieran. Las instrucciones de cómo responder se encontraban en las primeras pantallas; antes del primer reactivo del instrumento. Al término de la sesión se les agradeció su participación. Una vez aplicado el instrumento se procedió a recopilar los resultados por medio del módulo generador de resultados del editor de escalas versión 2.0 (Blanco, Ornelas, Tristán, et al., 2013). 
Tabla 1. Análisis descriptivos e índices de discriminación de los ítems del cuestionario "Cuestionario de estilos atributivos área de logros académicos". Muestra total.

\begin{tabular}{cccccc}
\hline Ítem & $\mathrm{M}$ & $\mathrm{DE}$ & $\mathrm{AS}$ & $\mathrm{CU}$ & $\mathrm{r}_{\mathrm{i} \text {-total }}$ \\
\hline Ítem 1 & 6.63 & 2.23 & -0.69 & 0.41 & .21 \\
Ítem 2 & 8.09 & 1.80 & -0.88 & 0.52 & .53 \\
Ítem 3 & 6.85 & 2.76 & -0.54 & -0.70 & .63 \\
Ítem 4 & 6.10 & 2.69 & -0.13 & -0.84 & .57 \\
Ítem 5 & 7.56 & 2.16 & -1.05 & 1.28 & .42 \\
Ítem 6 & 4.42 & 2.79 & -0.12 & -0.86 & -.34 \\
Ítem 7 & 5.93 & 2.64 & -0.07 & -0.73 & .48 \\
Ítem 8 & 7.30 & 2.62 & -0.64 & -0.57 & .66 \\
Ítem 9 & 7.66 & 2.00 & -0.72 & 0.25 & .48 \\
Ítem 10 & 7.64 & 1.77 & -0.53 & -0.12 & .52 \\
Ítem 11 & 6.63 & 2.47 & -0.66 & 0.11 & .22 \\
Ítem 12 & 4.37 & 2.72 & -0.06 & -0.84 & -.37 \\
Ítem 13 & 6.82 & 2.89 & -0.50 & -0.84 & .58 \\
Ítem 14 & 7.75 & 2.02 & -0.77 & 0.34 & .55 \\
Ítem 15 & 6.93 & 2.42 & -0.87 & 0.59 & .31 \\
Ítem 16 & 5.40 & 2.48 & 0.14 & -0.44 & .48 \\
Ítem 17 & 5.53 & 2.63 & 0.19 & -0.70 & .46 \\
Ítem 18 & 6.43 & 2.95 & -0.27 & -1.04 & .59 \\
Ítem 19 & 7.18 & 2.13 & -0.77 & 0.70 & .43 \\
Ítem 20 & 5.54 & 2.57 & 0.11 & -0.61 & .50 \\
Ítem 21 & 6.55 & 2.65 & -0.18 & -0.99 & .67 \\
Ítem 22 & 5.93 & 2.72 & -0.03 & -0.84 & .57 \\
Ítem 23 & 8.06 & 1.87 & -0.82 & 0.05 & .61 \\
Ítem 24 & 7.13 & 2.06 & -0.52 & 0.26 & .37 \\
Ítem 25 & 7.17 & 2.22 & -0.62 & 0.10 & .44 \\
Ítem 26 & 4.50 & 2.75 & -0.11 & -0.77 & -.27 \\
Ítem 27 & 5.00 & 2.67 & 0.22 & -0.57 & .30 \\
Ítem 28 & 6.34 & 2.43 & -0.63 & 0.31 & -.02 \\
Ítem 29 & 6.39 & 2.71 & -0.25 & -0.81 & .63 \\
Ítem 30 & 6.01 & 2.76 & -0.01 & -0.96 & .56 \\
Ítem 31 & 7.89 & 1.98 & -0.68 & -0.27 & .60 \\
Ítem 32 & 7.69 & 1.87 & -0.69 & 0.14 & .46 \\
Ítem 33 & 6.57 & 2.11 & -0.49 & 0.46 & .14 \\
Ítem 34 & 6.99 & 2.70 & -0.47 & -0.79 & .64 \\
Ítem 35 & 7.72 & 2.02 & -0.62 & -0.28 & .56 \\
Ítem 36 & 4.81 & 2.59 & 0.27 & -0.34 & .28 \\
Ítem 37 & 6.68 & 2.56 & -0.27 & -0.83 & .67 \\
Ítem 38 & 4.03 & 2.86 & 0.13 & -0.91 & -.33 \\
Ítem 39 & 5.36 & 2.76 & 0.11 & -0.69 & .38 \\
\hline & & & & & \\
\hline
\end{tabular}

\section{Análisis de datos}

El primer paso del análisis de las propiedades psicométricas del cuestionario consistió en calcular la media, la desviación estándar, la asimetría, la curtosis y los índices de discriminación de cada ítem. Para luego eliminar de la escala aquellos que obtienen una curtosis o asimetría extremas, o un índice de discriminación por debajo de 0.35 . Luego, se sometieron a comparación dos modelos de medida: el Modelo 1 (M1), modelo de 7 factores acorde a la distribución original de los ítems dentro del cuestionario y el Modelo 2 ( M2), que responde a una estructura tetra-factorial, eliminando los ítems que no fueron suficientemente bien explicados por ese modelo o que obtuvieron un índice de discriminación bajo y la fusión de los factores originales con correlaciones altas entre sí. 
Para conducir los análisis factoriales confirmatorios se utilizó el software AMOS 21 (Arbuckle, 2012), las varianzas de los términos de error fueron especificados como parámetros libres, en cada variable latente (factor) se fijó uno de los coeficientes estructurales asociados a uno, para que su escala sea igual a la de una de las variables observables (ítems). El método de estimación empleado fue el de Máxima Verosimilitud; siguiendo la recomendación de Thompson (2004), en el sentido de que cuando se emplea análisis factorial confirmatorio se debe corroborar no sólo el ajuste de un modelo teórico sino que es recomendable comparar los índices de ajuste de varios modelos alternativos para seleccionar el mejor.

Para evaluar el ajuste del modelo se emplearon el estadístico Chi-cuadrado, el índice de bondad de ajuste (GFI) y el error cuadrático medio de aproximación (RMSEA) como medidas absolutas de ajuste. El índice de bondad ajustado (AGFI), el Índice Tucker-Lewis (TLI) y el índice de bondad de ajuste comparativo (CFI) como medidas de ajuste incremental. La razón de Chi-cuadrado sobre los grados de libertad (CMIN/GL) y el Criterio de Información de Akaike (AIC) como medidas de ajuste de parsimonia (Byrne, 2010; Gelabert et al., 2011). Posteriormente, siguiendo las recomendaciones de Abalo et al. (2006), se llevó a cabo un análisis de la invarianza factorial del cuestionario para las submuestras tomando como base el mejor modelo de medida obtenido en la etapa anterior. Por último se calculó la fiabilidad de cada una de las dimensiones, de los modelos de medida obtenidos en cada submuestra, a través del Coeficiente Alpha de Cronbach (Elosua y Zumbo, 2008; Nunnally y Bernstein, 1995) y del Coeficiente Omega (Revelle y Zinbarg, 2009; Sijtsma, 2009).

\section{RESULTADOS Y DISCUSIÓN}

En la Tabla 1 se resumen los resultados de los análisis descriptivos y los índices de discriminación (correlación elemento-total corregida) de cada uno de los 39 ítems del cuestionario en la muestra total. En la Tabla 1 Nota: $\mathrm{M}=$ media; $\mathrm{DE}=$ desviación estándar; $\mathrm{AS}=$ =asimetría; $\mathrm{CU}=$ curtosis; $r_{\text {i-total }}=$ correlación elementototal corregida. Las respuestas a todos los ítems reflejan unas puntuaciones medias que oscilan entre 4.03 y 8.09, y la desviación estándar ofrece, en todos los casos, valores mayores a 1.5 (dentro de un rango de respuesta entre 0 y 10). Como se observa en la Tabla 1 , todos los valores de asimetría y curtosis se encuentran dentro del rango \pm 1.5 ; por lo que se infiere que las variables se ajustan razonablemente a una distribución normal. En cuanto a los índices de discriminación los ítems 6, 12, 26, 28, 33 y 38 no discriminan satisfactoriamente con índices de discriminación por debajo de .20 (Brzoska y Razum, 2010).

\section{Análisis factoriales confirmatorios}

Los resultados globales del análisis factorial confirmatorio en la submuestra 1 (GFI .821; RMSEA .064; CFI .862) y la submuestra 2 (GFI .807; RMSEA .067; CFI .843) para el modelo M1 que corresponde a la distribución original de los ítems dentro del cuestionario de estilos atributivos área de logros académicos, indican que el modelo de medición, en ambas submuestras se puede considerar como no aceptable (Tabla 2). En la Tabla, Nota: * $\mathrm{p}<$.05; GFI=índice de bondad de ajuste; RMSEA=raíz del error medio; AGFI=índice

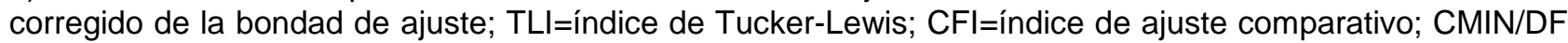
=índice de ajuste chi cuadrado dividido por los grados de libertad; AIC=criterio de información de Akaike

Tabla 2 Índices absolutos, incrementales y de parsimonia para los modelos generados. Submuestras 1 y 2.

\begin{tabular}{|c|c|c|c|c|c|c|c|c|c|c|}
\hline & \multicolumn{3}{|c|}{ Índices absolutos } & \multicolumn{3}{c|}{ Índices incrementales } & \multicolumn{2}{c|}{ Índices de parsimonia } \\
\hline Modelo & $\chi^{2}$ & GFI & RMSEA & AGFI & TLI & CFI & \multicolumn{2}{c|}{ CMIN/DF } & AIC \\
\hline \multicolumn{8}{|c|}{ Primera solución factorial (submuestra 1) } \\
\hline M1 & $2796.41^{*}$ & .821 & .064 & .795 & .850 & .862 & 4.100 & 2994.41 \\
\hline M2 & $525.177^{*}$ & .950 & .036 & .934 & .970 & .976 & 1.982 & 697.177 \\
\hline \multicolumn{8}{|c|}{ Segunda solución factorial (submuestra 2) } \\
\hline M1 & $2806.22^{*}$ & .807 & .067 & .779 & .829 & .843 & 4.120 & 3004.22 \\
\hline M2 & $572.651^{*}$ & .941 & .041 & .922 & .959 & .967 & 2.161 & 788.623 \\
\hline
\end{tabular}

El conjunto de los siete factores del modelo M1 explican aproximadamente el $60 \%$ de la varianza en ambas submuestras. Por otro lado 20 de los 39 ítems, en ambas submuestras, saturan por debajo de .70 en su dimensión prevista. Observándose además, intercorrelaciones altas entre los factores; Atribución del éxito académico a la buena suerte y Atribución del fracaso académico a la mala suerte; Atribución del fracaso académico a la falta de esfuerzo y Atribución del éxito al esfuerzo; Atribución del éxito académico a la habilidad y Atribución del fracaso a la falta de habilidad, evidenciando una pobre validez discriminante entre ellos. 
Los resultados globales del análisis factorial confirmatorio en la primer (GFI .950; RMSEA .036; CFI .976) y segunda submuestra (GFI .941; RMSEA .041; CFI .967), del segundo modelo sometido a prueba ( M2) que corresponde a una estructura tetradimensional del cuestionario, sin los ítems de más baja saturación en cada uno de los factores y la fusión de los factores con correlaciones altas entre sí, indican que este modelo de medición es mejor que el modelo anterior y que su ajuste es óptimo (Tabla 2). Los cuatro factores de este modelo explican en conjunto, en ambas submuestras aproximadamente el $60 \%$ de la varianza. Por otro lado de acuerdo a los resultados de la Tabla 3; solo tres de los 26 ítems en la primer submuestra y cinco en la segunda saturan por debajo de .60 en su dimensión prevista. Observándose además, intercorrelaciones de bajas a moderadas entre los factores evidenciando una adecuada validez discriminante entre ellos.

\section{Invarianza de la estructura factorial entre las submuestras}

Los índices de ajuste obtenidos (Tabla 4) permiten aceptar la equivalencia de los modelos de medida básicos entre las dos submuestras. Aunque el valor de Chi-cuadrado excede al requerido para aceptar la hipótesis de invarianza, los índices $\mathrm{GFI}=.946, \mathrm{CFI}=.972$, RMSEA=.027 y $\mathrm{AIC}=1441.832$ contradicen esta conclusión lo que nos permite aceptar el modelo base de la invarianza (modelo sin restricciones).

Añadiendo al modelo base restricciones sobre las cargas factoriales caracterizamos la invarianza métrica. Los valores que se recogen en la tabla 4 permiten aceptar este nivel de invarianza. El índice de ajuste general (GFI .945) y el error cuadrático medio de aproximación (RMSEA .027) siguen aportando información convergente en esta dirección. Además, el criterio de información de Akaike (AIC 1421.595) y el índice comparativo de Bentler (CFI .971) no sufren grandes variaciones respecto al modelo anterior.

Tabla 3 Soluciones estandarizadas análisis factorial confirmatorio para el Modelo M2. Submuestra 1 y 2 Nota: F1=atribución a la suerte, F2=atribución al esfuerzo, F3=atribución a la habilidad, F4=atribución al profesor.

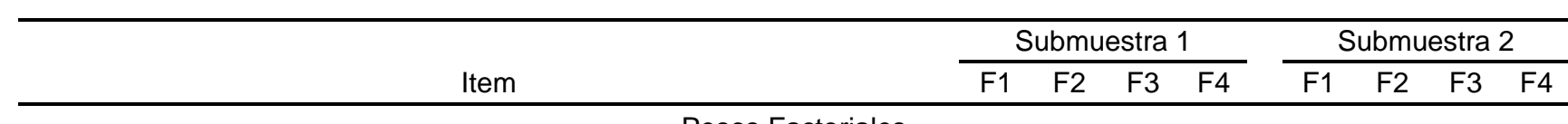

Pesos Factoriales

3. Por lo general, si he obtenido malos resultados en mis calificaciones $\quad .67$ creo que ha sido por mala suerte

8. Mi mala suerte ha sido muchas veces lo que hizo que no tuviera $\quad .74$ mejores calificaciones

13. La suerte ha sido, por lo general, la causa de mis buenas calificaciones en mis estudios

17. Frecuentemente mis bajas calificaciones en mis estudios se han debido, sobre todo, a equivocaciones casuales

18. La suerte es, con frecuencia, el principal factor responsable de mi éxito en los estudios

21. La casualidad ha sido la causa de que a veces haya tenido calificaciones bajas

22. Con frecuencia, si he sacado buenas calificaciones en una materia ha sido porque el profesor daba calificaciones altas con facilidad

30. Con frecuencia mis malas calificaciones se deben a la mala suerte de tocarme justamente las preguntas que no he preparado

34. Por lo general, apruebo simplemente por suerte

37. Me parece que, normalmente, mis calificaciones bajas se han debido a la casualidad

5. Si alguna vez he obtenido bajas calificaciones en mis estudios se ha debido a falta de esfuerzo

15. Las bajas calificaciones que he obtenido han sido, sobre todo, porque no me he esforzado lo suficiente

19. Normalmente, si recibo una mala nota en una materia es porque no he estudiado lo suficiente

2. En mi caso, sacar buenas calificaciones se ha debido, a mi propio esfuerzo

9. Siempre que he obtenido buenas calificaciones ha sido porque he estudiado mucho

.69

.76

.63

.68

.64

.55

.77

.75

.81

.82

.72

.70

.74

.67

.79

.75

.82

.75

.64

.54

.62

.50

.72

.76 
Tabla 3: Continuación.

10. Si saco buenas calificaciones es por mi buena capacidad para los estudios

14. Cuando he trabajado con empeño, generalmente he podido superar los obstáculos que me impedían tener éxito en los estudios

23. Si tengo buenas calificaciones en alguna materia es por el esfuerzo y empeño que pongo

24. Mi inteligencia constituye el factor más importante a la hora de conseguir buenas calificaciones

31. Cuando me he esforzado y he trabajado arduamente, he conseguido buenas calificaciones en mis estudios

2. Si alguna vez he obtenido buenos resultados se ha debido a mi capacidad para esa materia

35. Normalmente, cuando he trabajado arduamente he conseguido tener éxito en los estudios

7. Con frecuencia mis malas calificaciones se deben a que el profesor no está bien preparado para enseñarme

6. Si he tenido malas calificaciones en alguna materia a menudo ha sido porque el profesor explicaba mal

20. A menudo, si he tenido malas calificaciones ha sido porque los profesores no me han explicado bien las lecciones

39. Es frecuente, si saco malas calificaciones, que sea porque el profesor no ha hecho interesante la materia

Correlaciones Factoriales
\begin{tabular}{ccccccccccc} 
F1 & - & & & & - & & & \\
F2 & .22 & - & & & .36 & - & & \\
F3 & .57 & .67 & - & & .54 & .76 & - & \\
F4 & .71 & .08 & .26 & - & .69 & .24 & .23 & - \\
\hline
\end{tabular}

Haciendo uso del criterio para la evaluación de los modelos anidados propuesto por Cheung y Rensvold (2002), quiénes sugieren que si el cálculo de la diferencia de los CFI de ambos modelos anidados disminuye en .01 o menos, se da por bueno el modelo restringido y por tanto el cumplimiento de la invarianza factorial; la diferencia entre CFIs obtenida permite aceptar el modelo de invarianza métrica. Podemos concluir hasta ahora que las cargas factoriales son equivalentes en las dos submuestras.

Una vez demostrada la invarianza métrica entre las submuestras, pasamos a evaluar la equivalencia entre interceptos (invarianza factorial fuerte). Los índices (Tabla 4) muestran un ajuste aceptable de este modelo, tanto evaluado de modo independiente como analizándolo respecto a su anidamiento con el modelo de invarianza métrica. En la Tabla 4, Nota: * $p<.05$; GFI = índice de bondad de ajuste; NFI = índice de ajuste normado; CFI = índice de ajuste comparativo; RMSEA = raíz del error medio; AIC = criterio de Información de Akaike. La diferencia entre los índices comparativos de Bentler es de .001; el índice de ajuste general es .944 y el error cuadrático medio de aproximación es .027. Aceptada la invarianza fuerte, los dos modelos evaluados son equivalentes respecto a los coeficientes factoriales y a los interceptos.

Tabla 4 Índices de bondad de ajuste de cada uno de los modelos puestos a prueba en la invarianza factorial.

\begin{tabular}{|l|c|c|c|c|c|c|c|}
\hline \multicolumn{1}{|c|}{ Modelo } & \multicolumn{7}{c|}{ Índice de Ajuste } \\
\hline & $\chi^{2}$ & gl & GFI & NFI & CFI & RMSEA & AIC \\
\hline Modelo sin restricciones & $1097.832^{*}$ & 530 & .946 & .947 & .972 & .027 & 1441.832 \\
\hline Invarianza métrica & $1121.595^{\star}$ & 552 & .945 & .946 & .971 & .027 & 1421.595 \\
\hline Invarianza factorial fuerte & $1146.790^{*}$ & 562 & .944 & .944 & .970 & .027 & 1426.790 \\
\hline
\end{tabular}

Los factores obtenidos en los análisis factoriales confirmatorios alcanzan, en su mayoría valores de consistencia interna por encima de .75 en ambas submuestras; evidenciando una consistencia interna adecuada para este tipo de subescalas, particularmente si se considera el número reducido de ítems (Tabla 5). 
Tabla 5. Coeficiente omega y alfa para los factores obtenidos en los análisis factoriales exploratorios submuestras 1 y 2 .

\begin{tabular}{|l|c|c|c|c|}
\hline & \multicolumn{2}{|c|}{ Submuestra 1 } & \multicolumn{2}{c|}{ Submuestra 2 } \\
\hline Factor & $\Omega$ & $\alpha$ & $\Omega$ & $\alpha$ \\
\hline atribución a la suerte & .921 & .925 & .912 & .917 \\
\hline atribución al esfuerzo & .700 & .722 & .631 & .682 \\
\hline atribución a la habilidad & .873 & .888 & .889 & .899 \\
\hline atribución al profesor & .851 & .848 & .796 & .787 \\
\hline
\end{tabular}

El objetivo principal del estudio fue indagar si se replican o no los resultados psicométricos propuestos por Matalinares et al. (2009) para el cuestionario de estilos atributivos en el área de logros académicos a través de una muestra de universitarios utilizando el análisis factorial confirmatorio. Los análisis factoriales confirmatorios realizados en cada submuestra por separado apoyan una estructura factorial de cuatro factores: atribución a la suerte, atribución al esfuerzo, atribución a la habilidad y atribución al profesor al evidenciar una consistencia interna adecuada, particularmente si se considera el número reducido de ítems en cada uno de ellos; al mismo tiempo que los factores así obtenidos presentan en general saturaciones factoriales estandarizadas adecuadas. Sugiriendo además la existencia de fuertes evidencias de la validación cruzada de la medida y por tanto de la estabilidad de la estructura hasta que no se demuestre lo contrario. Sin embargo el modelo obtenido no coincide con el planteado por Matalinares et al. (2009), ya que para lograr un mejor ajuste y una mayor capacidad de discriminación hubo que eliminar 13 de los 39 ítems analizados y cambiar la saturación original de algunos ítems; esto último con base en los índices de modificación y a su justificación teórica. Las discrepancias observadas entre el modelo propuesto por Matalinares et al. (2009) y el aquí formulado pueden atribuirse a diferencias sociales o culturales de los participantes; como el de ser estudiantes universitarios del área de la actividad física.

En síntesis, el análisis de las propiedades psicométricas del cuestionario de estilos atributivos en el área de logros académicos, ha mostrado que una estructura tetrafactorial es viable y adecuada de acuerdo a los requisitos psicométricos establecidos. La estructura de cuatro factores, atendiendo a criterios estadísticos y sustantivos, ha mostrado adecuados indicadores de ajuste, de fiabilidad y de validez. Sin embargo, el alcance de estos resultados es limitado, y es necesario que en investigaciones futuras se confirme la estructura obtenida, lo cual permitirá contar con evidencia más robusta respecto a la estructura factorial de la escala. Específicamente, debe demostrarse si la invarianza de la estructura de la escala se cumple por género, edad, entre alumnos de distintas licenciaturas, entre otras; de tal manera que, se considera que más estudios son necesarios con el fin de corroborar o refutar los datos obtenidos en las investigaciones realizadas hasta el momento. Asimismo, es indispensable comprobar si la escala resulta útil para estudiar la relación entre los estilos atributivos y el aprendizaje.

\section{CONCLUSIONES}

Con base en el estudio presentado, se pueden extraer las siguientes conclusiones principales:

i) El Análisis Factorial Confirmatorio, en ambas submuestras, indicó que el ajuste de los datos al modelo teórico de 26 reactivos agrupados en cuatro factores es aceptable.

ii) Los factores en ambas submuestras evidenciaron una consistencia interna adecuada, particularmente si se considera el número reducido de reactivos en cada uno de ellos

iii) Los resultados del análisis de la invarianza factorial entre las submuestras; indican una alta congruencia entre pares de factores.

iv) Cuando menos dos limitaciones están presentes en este trabajo. La primera es que los participantes son solo estudiantes del área de la actividad física, lo que supone una amenaza para la posibilidad de generalizar estos resultados. Ampliar la muestra (agregando por ejemplo adultos jóvenes o adolescentes que no son estudiantes) es un área de trabajo de cara al futuro. La segunda limitación proviene del propio instrumento de evaluación, que se basa en el autoinforme y que puede contener los sesgos que se derivan de la deseabilidad social.

\section{REFERENCIAS}

Abalo J., Lévy J., Rial A. y Varela J., Invarianza factorial con muestras múltiples, En Modelización con Estructuras de Covarianzas en Ciencias Sociales por J. Lévy, pp 259-278 Netbiblo, Madrid, (2006) 
Alonso J. y Sánchez J. C., Estilos atributivos y motivación: el cuestionario EAT, En Motivar en la adolescencia: Teoría, evaluación e intervención, por J. A. Tapia, pp 93-133 Madrid: Ediciones de la Univesrsidad Autónoma, D. L., (1992)

Arbuckle J. R., AMOS users guide version 21.0, Marketing Department, SPSS Incorporated, Chicago, IL, (2012)

Blanco H., Ornelas M., Rueda M. B. y Martínez M., Composición factorial de la escala de autoeficacia en conductas académicas en universitarios de ciencias sociales, Revista Mexicana de Psicología, 30(1), 79-88, (2013)

Blanco H., Ornelas M., Tristán J. L., Cocca A., Mayorga-Vega D., López-Walle J., et al., Editor for creating and applying computerise surveys, doi: http://dx.doi.org/10.1016/j.sbspro.2013.12.105, Procedia Social and Behavioral Sciences, 106, 935-940, (2013)

Brenlla M. y Vázquez N., Análisis Psicométrico de la adaptación argentina de la escala de Locus de Control de Rotter, 2, 1-33, 2010.

http://www.uca.edu.ar/uca/common/grupo68/files/Documento_de_Trabajo_Locus_de_Control1.pdf.

Brzoska P. y Razum O., Validity Issues in Quantitative Migrant Health Research: The Example of IIIness Perceptions, Peter Lang International Academic Publishers, New York, NY, (2010)

Byrne B. M., Structural Equation Modeling With AMOS: Basic Concepts, Applications, and Programming, Routledge, New York, NY, (2010)

Camgoz S. M., Tektas O. O. y Metin I., Academic attributional style, self-efficacy and gender: A crosscultural comparison, Social Behavior and Personality: an international journal, 36(1), 97-114, (2008)

Cheung G. W. y Rensvold R. B., Evaluating goodness-of-fit indexes for testing measurement invariance, doi: 10.1207/s15328007SEM0902_5, Structural Equation Modeling, 9(2), 233-255, (2002)

Durán-Aponte E. y Pujol L., Diferencias de género y área de estudio en las atribuciones causales de estudiantes universitarios, Anales de la Universidad Metropolitana, 12(2), 39-51, (2012)

Durán-Aponte E. y Pujol L., Escala Atribucional de Motivación de Logro General (EAML-G): Adaptación y análisis de sus propiedades psicométricas, Estudios Pedagógicos, 39(1), 83-97, (2013)

Elosua P. y Zumbo B. D., Coeficientes de fiabilidad para escalas de respuesta categórica ordenadas, Psicothema, 20(4), 896-901, (2008)

Fernández, A., Arnaiz, P., Mejía, R., \& Barca, A., Atribuciones causales del alumnado universitario de República Dominicana con alto y bajo rendimiento académico, doi: 10.17979/reipe.2015.2.1.1319, Revista de Estudios e Investigación en Psicología y Educación, 2(1), 19-29, (2015)

Ferrando P. J., Demestre J., Anguiano-Carrasco C. y Chico E., Evaluación TRI de la escala I-E de Rotter: un nuevo enfoque y algunas consideraciones, Psicothema, 23(2), 282-288, (2011)

Gelabert E., García-Esteve L., Martín-Santos R., Gutiérrez F., Torres A. y Subirà S., Psychometric properties of the Spanish version of the Frost Multidimensional Perfectionism Scale in women, Psicothema, 23(1), 133139, (2011)

Inglés C. J., Díaz-Herrero Á., García-Fernández J. M., Ruiz-Esteban C., Delgado B. y Martínez-Monteagudo M. C., Auto-atribuciones académicas: Diferencias de género y curso en estudiantes de educación secundaria, Revista Latinoamericana de Psicología, 44(3), 57-68, (2012)

Inglés C. J., Rodríguez-Marín J. y González-Pienda J. A., Adaptación de la Sydney Attribution Scale en población universitaria española, Psicothema, 20(1), 166-173, (2008)

Matalinares M., Tueros R. y Yaringaño J., Adaptación psicométrica del Cuestionario de Estilos Atributivos, Revista de Investigación en Psicología, 12(1), 173-189, (2009)

Mkumbo K. A. K. y Amani J., Perceived university students' attributions of their academic success and failure, doi: 10.5539/ass.v8n7p247, Asian Social Science, 8(7), 247-255, (2012)

Montero I. y León O., A guide for naming research studies in psychology, International Journal of Clinical and Health Psychology, 7(3), 847-862, (2007) 
Nunnally J. C. y Bernstein I. H., Teoría Psicométrica, McGraw-Hill, México, (1995)

Revelle W. y Zinbarg R. E., Coefficients alpha, beta, omega and the glb: comments on Sijtsma, doi: 10.1007/s11336-008-9102-z, Psychometrika, 74(1), 145-154, (2009)

Richardson M., Abraham C. y Bond R., Psychological correlates of university students' academic performance: A sytematic review and meta-analysis, doi: 10.1037/a0026838, Psychological Bulletin, 138(2), 353-387, (2012)

Salvador C. y Mayoral L., Influencia de los estilos de atribución en la inteligencia emocional de los estudiantes argentinos, Revista Electrónica Interuniversitaria de Formación del Profesorado, 14(1), 243-251, (2011)

Sanjuán P. y Magallares A., Estilo atributivo negativo, sucesos vitales y sintomatología depresiva, Revista de psicopatología y psicología clínica, 11(2), (2006)

Sijtsma K., On the use, the misuse, and the very limited usefulness of Cronbach's alpha, doi: 10.1007/s11336-008-9101-0, Psychometrika, 74(1), 107-120, (2009)

Soria-Barreto K. y Zúñiga-Jara S., Aspectos determinantes del éxito académico de estudiantes universitarios, doi: 10.4067/S0718-50062014000500006, Formación Universitaria, 7(5), 41-50, (2014)

Tavakolizadeh J. y Ebrahimi S., El efecto de enseñar estrategias de aprendizaje autorregulado sobre estilos de atribución de los alumnos, Electronic Journal of Research in Educational Psychology, 9(25), 1087-1101, (2011)

Thompson B., Exploratory and Confirmatory Factor Analysis. Understanding concepts and applications. , American Psychological Association, Washington, D C, (2004)

Viciana J., Cervelló E. M., Ramírez J., Effects of manipulating positive and negative feedback on goal orientation, perceived motivational climate, satisfaction, task choice, perception of ability, and attitude to physical education lessons, Perceptual and motor skills, 105(1), 67-82, (2007)

Weiner B., Intrapersonal and interpersonal theories of motivation from an attributional perspective, Educational Psychology Review, 12(1), 1-15, (2000)

Weiner B., Attribution theory revisited: Transforming cultural plurality into theoretical unity, En Big Theories Revisited, por D. M. McInerney, Van Etten S., pp 13-30 Information Age Pub., (2004) 